\title{
CONTRIBUCIÓN DE LA OBRA SOCIAL FEMENINA DE NUESTRA SEÑORA DE LOS DESAMPARADOS AL DESARROLLO DE LA ENFERMERÍA EN LA VALENCIA DE LOS AÑOS CUARENTA
}

\author{
María Ángeles Reig Ureña \\ Universidad Católica de Valencia San Vicente Mártir \\ Email: reurma@gmail.com \\ ORCID iD: http://orcid.org/0000-0001-6161-8144 \\ Francisco Abelardo Cardells Martí \\ Universidad Católica de Valencia San Vicente Mártir \\ Email: fa.cardells@ucv.es \\ ORCID iD: http://orcid.org/0000-0002-4194-2294
}

Recibido: 24 febrero 2020; Aceptado: 28 octubre 2020

Cómo citar este artículo/Citation: Reig Ureña, María Ángeles; Cardells Martí, Francisco Abelardo (2021) “Contribución de la obra social femenina de Nuestra Señora de los Desamparados al desarrollo de la enfermería en la Valencia de los años cuarenta", Asclepio, 73(1): p346. https://doi.org/10.3989/asclepio.2021.12

RESUMEN: Atender a las particularidades de las diversas disciplinas profesionales contribuye a enriquecer el corpus de conocimientos que las integran. Las aportaciones que se realizan desde el ámbito local ayudan a completar este conocimiento y ponen de manifiesto la singularidad de unos acontecimientos que, desencadenados por diversos condicionantes, explican el hecho histórico.

El objetivo perseguido con la redacción de este artículo se centra en dar a conocer la influencia que, la iniciativa llevada a cabo por la Obra Social Femenina de Nuestra Señora de los Desamparados de Valencia (OSF) tuvo en la promoción personal y profesional de la mujer valenciana. Concretamente centramos nuestra atención en la tarea llevada a cabo desde la OSF en la promoción de la mujer enfermera.

Siguiendo el modelo de investigación histórico basado en la obtención de los datos de forma organizada y sistemática, consultamos fuentes primarias que aportan datos relevantes y hasta hoy desconocidos al estudio y consultamos también bibliografía especializada que nos ayuda a situar, analizar y comprender las circunstancias en las que acontecen los hechos. Enfatizando en el rol social que jugaría la mujer durante las primeras décadas del franquismo y justificando la necesidad de profesionales sanitarios que velaran por poner en práctica las medidas eugenésicas propuestas por el Nuevo Régimen, procuraremos una visión general de la profesión enfermera en aquellos años. Para terminar, y en el contexto de una de las instituciones privadas, que al amparo de la orden de 21 de mayo de 1941 ofrecía la posibilidad de dar validez oficial a los estudios de enfermera, llegamos a descubrir la presencia de una agrupación local de enfermeras que impulsada desde las filas del asociacionismo confesional de principios de siglo XX, contribuyó a la formación de las enfermeras valencianas.

PALABRAS CLAVE: Formación enfermera; Valencia; Obra Social Femenina (OSF); Salus Infirmorum; Sindicato confesional femenino. 
ABSTRACT: Attending to the particularities of the various professional disciplines helps to enrich the body of knowledge that integrates them. The contributions made from the local level help complete this knowledge and highlight the uniqueness of some events that, triggered by various conditions, explain the historical fact.

The objective pursued with the writing of this article focuses on making known the influence that the initiative carried out by the Obra Social Femenina de Ntra. Sra. de los Desamparados de Valencia (OSF) had on the personal and professional promotion of women Valencian. Specifically, we focus our attention on the task carried out by the OSF in the promotion of women nurses.

Following the historical research model based on obtaining the data in an organized and systematic way, we consult primary sources that provide relevant data and until now unknown to the study and also consult specialized bibliography that helps us locate, analyze and understand the circumstances in the that the events happen. Emphasizing the social role that women would play during the first decades of Franquismo and justifying the need for health professionals to ensure the implementation of the eugenic measures proposed by the new Regime, we will seek an overview of the nursing profession in those years. Finally, and in the context of one of the private institutions, which under the order of May 21, 1941 offered the possibility of giving official validity to nursing studies, we discovered the presence of a local group of nurses which, promoted from the ranks of the confessional association of the early twentieth century, contributed to the training of Valencian nurses.

KEY WORDS: Nurse Training; Valencia; Obra Social Femenina (OSF); Salus Infirmorum; Women's Confesional Unión

Tras el conflicto bélico, con la llegada del nacional-catolicismo y la eclosión de la Sección Femenina de FET y JONS como elementos predominantes en el control ideológico de la población, especialmente de la femenina, se iniciaría una etapa marcada por el resurgimiento de la presencia de órdenes religiosas en la atención al enfermo, la división del trabajo en función de los sexos o la aparición de un sistema formativo con claras connotaciones ideológicas de dudosa calidad formativa (Siles González, 2008, p. 284).

Las enfermeras, ávidas de un reconocimiento formal e institucional de sus funciones desde su reconocimiento como profesión en 1915, encontrarán en estos años difíciles una oportunidad para su desarrollo y promoción profesional. Su imagen ligada al estereotipo propuesto por el estado como ideal de mujer y la patente división de roles respecto al género que impregnarán la esfera social, a la que sumamos las difíciles situaciones de pobreza, insalubridad y enfermedad surgidas como consecuencia del conflicto bélico, supondrán una oportunidad para visibilizar la función de las enfermeras sobretodo en el ámbito comunitario.

Si las mujeres tuvieron proyección político social y cierta autonomía en la educación y en las políticas asistenciales durante el periodo franquista, fue porque interviniendo en estos campos lograban resaltar y acentuar su misión como educadoras y madres de familia. Si durante la segunda República, algunos grupos de católicas activistas habían alzado la voz y se habían manifestado en aras de una educación católica, en defensa de la familia tradicional y en la necesidad de mantener a la mujer en el hogar para perpetuar el modelo de familia tradicional y cristiana que se veía altamente amenazado por la irrupción de nuevas ideologías liberales y marxistas, ahora con la implantación del Nuevo Régimen continuarían luchando por la defensa de estos valores pero motivadas por otros como, la regeneración nacional o el ensalzamiento de la Patria.

La preocupación pronatalista del Régimen, sintetizada en la famosa frase del Caudillo de llegar a la cifra de "cuarenta millones de españoles", incluía también medidas encaminadas a la educación de las madres en principios de puericultura e higiene, pues serán ellas las encargadas de "suministrar a la Patria hombres para engrandecerla" (Sánchez Blanco, 2010, p. 227-236; Cayuela Sánchez, 2014, p. 202). Esta idea, se asentará sobre un discurso basado en la re-conceptualización de la maternidad como un deber social femenino, y en el ejercicio de la maternidad como una misión casi profesional. Así, el cometido social de las mujeres será garantizar la procreación y la supervivencia de las futuras generaciones de ciudadanos en óptimas condiciones de salud e higiene (Nash 2000, p. 693), pasando a ser disciplinas como la puericultura y la maternología elementos clave de la política demográfica franquista, y la tarea de instruir y educar a la mujer en sus deberes maternales un objetivo claro (Palacio Lis 2003, p. 196-242).

La política sanitaria de los primeros años del franquismo, se centró en dos cuestiones primordiales, aumentar la natalidad y disminuir la mortalidad infantil. 
Para ello, las nociones sobre cuidados en cuestiones de puericultura e higiene tenían que ser llevadas a todos los rincones de la geografía española, y figuras como las divulgadoras rurales de la Sección Femenina, las instructoras de sanidad y las enfermeras sociales surgirían con fuerza para poder transmitir estas cuestiones hasta los pueblos más remotos (Bernabeu Mestre y Gascón Pérez 1999, p. 102-109). El oficio de enfermera se alzaba como una "pieza esencial en la medicina social del franquismo, al ser utilizado como correa de transmisión de las prácticas higiénico-sanitarias impulsadas por la Sección Femenina" (Fernández Giménez 2008, p. 231).

Además, la enfermera encarnaba a la perfección el modelo de mujer defendido por el franquismo, tanto es así, que entre las carreras aptas o apropiadas a las que las mujeres podían dedicarse se encontraba la de enfermera (Agulló Diaz 1999, p. 251).

La asistencia sanitaria, la educación o el control de la salubridad e higiene serían tareas llevadas a cabo por mujeres, por lo que la demanda de estas nuevas profesionales fue en aumento.

Por otro lado, la marcada superpoblación en algunas ciudades y el hacinamiento consecuente, actuaron como factores favorecedores de la aparición de tuberculosis y otras enfermedades como el tifus exantemático, viruela, paludismo, avitaminosis, anemias y difteria, que componían el catálogo de las enfermedades recurrentes en aquellas décadas de miseria y condiciones higiénicas deplorables (García Ferrandis 2013, p.13-34). Estas situaciones que requerían de un control sanitario urgente junto con otras motivaciones de orden ideológico como fue la difusión de la ideología del Régimen, fueron tareas de las que la mujer fue máxima responsable.

La dispersión de figuras encargadas de poner en poner en práctica las políticas sanitarias del franquismo, así como los diferentes programas formativos que las acreditaban o el ámbito profesional donde desempeñaban su función fue tan variado e irregular que provocó la aparición de varias figuras profesionales con unos cometidos de gran similitud.

Mostramos a continuación una breve reseña de la situación en la que se encontraba la profesión enfermera al finalizar la contienda.

Cuando en julio del 1936 estalló la guerra civil, tanto en el bando republicano como en el sublevado, muchas mujeres se ofrecieron como voluntarias para asistir a los heridos, incluso careciendo de experiencia en cuestiones sanitarias. Así, dentro del Bando Nacional, Cruz Roja, las Margaritas del partido carlista, la Sección femenina de la Falange y Sanidad Militar, fueron los 4 organismos que ofrecieron formación para la asistencia sanitaria (López Vallecillo, 2016 p. 419-439). El Frente Popular estuvo apoyado por organizaciones feministas tales como la Unión de Mujeres Antifascistas, el Socorro Rojo Internacional, las Brigadas Internacionales, las Escuelas de Alerta y el Comité Internacional de Cruz Roja (Domínguez Isabel, et al. 2019, p. 77-86).

Al terminar la guerra, la necesidad de implementación de las nuevas políticas sanitarias y la gran diversidad de figuras encargadas de la atención sanitaria no estrictamente médica, exigía una reglamentación profesional que las aunara. Y aunque no todas corrieron la misma suerte, - pues los títulos expedidos durante la guerra, en zona republicana, fueron anulados al terminar ésta (Domínguez Isabel, et al. p. 83), - en 1942 se creó el Cuerpo de Enfermeras de Falange Española Tradicionalista y de las J.O.N.S. que daba cabida a todas las enfermeras de distinta formación que tras validar su título y completar su formación con los cursos pertinentes, podían especializarse en enfermeras de guerra y/o visitadoras sociales (BOE, 13, p. 244).

Además de las enfermeras de Falange Española, en 1941 se inauguraría la Escuela Nacional de Instructoras Sanitarias, dependiente de Sanidad Nacional, donde se formaría a las futuras instructoras de sanidad, que pertenecerían al Patronato Nacional Antituberculoso y enfermedades del tórax o se especializarían en puericultura. (Cantero González, Hernández Conesa y Beneit Montesinos, 2010, p.287-291).

Del Ministerio de Gobernación, concretamente de la Dirección General de Beneficencia, dependerían las enfermeras del Auxilio Social, que solapaban sus funciones con las de las visitadoras de la Sección Femenina o las de las instructoras de la Escuela Nacional de Sanidad (Bernabeu Mestre y Gascón González, 1999, p.105-106). Éstas, debían cumplir con una serie de requisitos que poco tenían que ver con nociones técnicas o profesionales,

"Deben ser simpáticas, inteligentes y con gran tacto social, cariñosas con los niños, abnegadas, capaces de tener influencia con las madres y nodrizas, y someterse $\sin$ vacilaciones a las órdenes del Médico asesor provincial". ${ }^{1}$

Y por último, el cuerpo de Divulgadoras Sanitarias que surgía desde la Hermandad de la Ciudad y del Campo, se encargaría de la atención sanitaria en las zonas rurales (Bernabeu Mestre y Gascón González, 1999, p.102-109). 
A estas profesionales, se sumaban los practicantes y matronas formados en las facultades de medicina desde 1857 en Madrid, Barcelona, Valencia, Sevilla, Cádiz, Granada, Salamanca, Santiago, Valladolid y Zamora, las enfermeras que eran preparadas por las Escuelas de la Cruz Roja española que distinguía entre las profesionales y las damas auxiliares (Camaño Puig y Forero Rincón, 1998, p. 161), y las órdenes religiosas, que seguían prestando cuidados en el ámbito hospitalario. Las Hijas de la Caridad de San Vicente de Paúl o los Hermanos de la Orden Hospitalaria de San Juan de Dios, constituyen dos claros ejemplos de ello (Cantero González, Hernández Conesa y Beneit Montesinos, 2010, p.255).

Otras iniciativas de carácter privado, impulsadas desde las filas del asociacionismo confesional y con una importante repercusión a nivel nacional, también trabajarán por la formación y promoción de las enfermeras. La Hermandad Profesional de enfermeras Salus Infirmorum, constituida en el seno de la Acción Católica y fundada por María de Madariaga en Madrid, además de contribuir a la formación de futuras enfermeras a través de su escuela inaugurada en 1943, ofrecerá a las enfermeras y futuras enfermeras una oportunidad para organizarse profesionalmente y luchar en favor de los intereses humanos y profesionales de sus componentes. La Hermandad aglutinará a enfermeras representantes de Renfe, Sanidad Militar, Sanidad Civil, del Seguro de Enfermedad, de la Obra de 18 de julio, Cruz Roja, Auxilio Social, de residencias sanitarias, de clínicas y sanatorios y a las voluntarias de servicios parroquiales de suburbios, pues en aquellos momentos no existe ningún colegio o asociación profesional de enfermeras que las ampare (Chamorro Rebollo, 2016, p.122). Será la orden de 1945 por la que se aprueba el Estatuto de los Colegios de Practicantes, Matronas y Enfermeras, el primer intento de unificación colegial y profesional en la historia de la profesión (BOE 293, p. 3334-3343).

A nivel local, y concretamente en la ciudad de Valencia, también surgirá una iniciativa a favor de la mujer enfermera que luchará por su promoción personal y profesional. Capitaneada por Ana Pons Carda, la agrupación de enfermeras de la Obra Social Femenina de Ntra. Sra. de los Desamparados, contribuirá durante los años cuarenta a la formación de las enfermeras valencianas.

En este trabajo, se pretende evaluar la situación de la formación enfermera en la posguerra, la influencia de las políticas sanitarias del primer franquismo en la sociedad y la relación entre estas políticas y la cuestión del género, con la intención de establecer relaciones que expliquen el devenir de la profesión.

La consulta de fuentes primarias localizadas en el Achivo de la Obra Social Femenina de Ntra. Sra. de los Desamparados de Valencia, situada en la calle Serrans número 3 , nos ha permitido acceder a una documentación novedosa e inédita, que completa la historia de la profesión enfermera, sobre todo a nivel local.

Desde mediados del siglo XIX, se suceden en España una serie de cambios socioeconómicos que suscitarán el nacimiento de nuevos movimientos sociales. El liberalismo económico, impulsó el desarrollo industrial en zonas como Cataluña o el País Vasco (Raveux, 2005, p. 157-184; Escudero Gutiérrez, 1999, p.191-200). El despegue de la explotación minera en el norte del país favoreció la aparición de la empresa siderurgia, los altos hornos fueron sustituidos por las antiguas herrerías y el sistema de producción organizado en fábricas fue ganando terreno. Los nuevos modelos de producción exigían cambios sociales y demográficos que no tardaron en tener consecuencias en la vida de la nueva clase social surgida: el proletariado.

Las fábricas se van a instalar en las ciudades lo que provocará un trasvase de mano de obra y recursos desde la agricultura a la industria. Estos núcleos de población registrarán un importante crecimiento con los problemas inherentes a la masificación. Hacinamiento, pobreza, malas condiciones en la vivienda, humo de las chimeneas de las fábricas, basura, poca sanidad y la tensión entre los obreros proletarizados y los capitalistas serán problemas cada vez más frecuentes (Chaves Palacios, 2004, p. 98).

De este modo, la asociación entre iguales en aras de luchar por unos derechos laborales dignos y justos, así como la demanda a las autoridades de jurados mixtos para negociar colectivamente las condiciones laborales o la reivindicación a través de prensa escrita para concienciar a la sociedad, tomarán todo su sentido, surgiendo así el conocido como movimiento obrero.

También desde la Iglesia católica, se pondrán en marcha iniciativas a favor de los más desfavorecidos en este nuevo orden social. El conocido catolicismo social alentado por las directrices expuestas en la encíclica Rerum Novarum de León XIII, sobre las condiciones de las clases trabajadoras, ofrecerá una visión del obrero como persona de derechos y merecedor de un sustento que le garantice la posibilidad de vivir una vida digna (León XIII, 1891). 
A partir de esta nueva concepción de la intervención social de la Iglesia que Montero describe como un proceso de maduración desde una mentalidad tradicional benéfico- caritativa hacia una actitud nueva, propiamente social (Montero García, 1984, p. 187) se pondrán en marcha iniciativas a favor de obreros y trabajadores.

En este contexto de lucha entre clases y reivindicación de derechos socio- laborales, la situación de los obreros se auguraba difícil y precaria, pero aún peor resultaría para la mujer. (Scott, 1993, pp. 427461; Arbiza Villalonga, 2000, pp. 395-458). El trabajo extra doméstico se impuso para ellas como un deber inexcusable, su sustento y el de su familia dependía ya no solamente del trabajo del hombre sino del suyo propio, de este modo, el trabajo femenino se convertía en una amenaza que hacía tambalearse los pilares sobre los que se sustentaba la familia tradicional. Esto provocó una actitud de rechazo hacia el trabajo de la mujer, especialmente el de la mujer casada en un taIler o fábrica fuera del domicilio familiar. Sus salarios eran mucho más bajos que los de los hombres, no era contemplada la baja por maternidad y su pluri- actividad en el hogar y en el trabajo las llevaba a jornadas extenuantes que desde muy jóvenes mermarían su salud, más si tenemos en cuenta los múltiples partos que sufrían (Monlleó Peris 2004, p. 133). El trabajo desempeñado por las mujeres tenía un carácter subsidiario respecto al del hombre, constituía junto con los niños, mano de obra barata, sin cualificar y sin posibilidades de promoción y ascenso a nivel profesional. La falta de instrucción, la educación basada en la pervivencia de los roles sexuales tradicionales, y el cultivo de las virtudes de abnegación y obediencia que se le suponían a la mujer, acallaban sus posibles reivindicaciones y hacían del empleo femenino una sustanciosa opción para los empleadores. Mucho más aceptado y conveniente se vio el trabajo a domicilio, que permitía a las mujeres atender a sus obligaciones domésticas, pero la realidad vivida por las mujeres que lo desempañaron fue bien distinta. Las largas jornadas de trabajo, los sueldos insuficientes junto con la desprotección legal, hicieron de la obrera de la aguja "víctima de unas condiciones de vida y de trabajo extremadamente duras e injustas, necesitada de ayuda para mejorar su situación y orientar adecuadamente su vida" (Blasco Herranz, 2008, p. 242).

En el caso de Valencia, la ocupación de las mujeres valencianas y su entorno próximo, se centraba básicamente en actividades agrarias y en actividades relacionadas con la industria textil y la indumentaria, sin olvidar la cerámica y la industria tabaquera (Teixidor de Otto y Hernández Soriano, 1998, p. 339-354).

Junto con Madrid y Barcelona, Valencia capitaneaba la lista de ciudades en las que mayor número de mujeres se dedicaban a la industria del vestido. En 1914 , se dedican a la industria del tocado y vestido en Valencia 16.853 mujeres, mientras que en 1920 y 1925 , las obreras valencianas dedicadas al vestido y tocado, ascendían a 18.525 y 18.973 respectivamente (Capel, 1986, p.161).

Ante la difícil situación que sufre la mujer obrera, empiezan a surgir voces que reivindican para ellas mejoras en las condiciones laborales y sociales.

El 12 de mayo de 1912 y a iniciativa de Don Manuel Pérez Arnal, sacerdote y canónigo de la catedral de Valencia, quedaría legalmente constituido un sindicato católico femenino que contó en sus inicios con 19 socias (Ramón Fernández, 2001, p. 87). El Sindicato obrero de trabajadoras de la Aguja y similares, bajo la protección de Nuestra Señora de los Desamparados, que fue el nombre que adoptó la agrupación femenina, prorrumpía con tres objetivos principales: El estudio, defensa y desarrollo de los intereses morales y profesionales de las asociadas, la mejora económica de las asociadas mediante instituciones de asistencia y previsión y finalmente, la instalación de oficinas de dirección profesional para atender las cuestiones derivadas del desempeño laboral de las obreras².

Este sindicato, surgido desde la sección de lecturas de la Obra de Protección de Intereses Católicos, obra adherida a la Acción Católica de la Mujer, proponía en el artículo 44 de sus estatutos, la clasificación de los diferentes oficios por gremios profesionales y éstos divididos por secciones o grupos de parroquias, con su Junta Directiva compuesta de Presidencia, Secretaria y Tesorera con sus vices o auxiliares y de las Delegadas parroquiales, elegidas todas entre las sindicadas del mismo oficio ${ }^{3}$.

Alrededor de este sindicato, legalmente constituido, e instalado inicialmente en el entresuelo de la calle Hierros de la Ciudad, 2, se agruparon los distintos gremios de mujeres trabajadoras: Modistas, Costureras, Sastreras, Ropa Blanca, Bordadoras, Sombrereras, Pasamaneras, Calceteras, Cajas de Cartón, Corseteras, Fábrica e Industria y del Arte de la Seda, a los cuales se unieron más tarde, los de Dependientas y el de Sirvientas (Palacio Lis y Ruiz Rodrigo, 1990, p. 651).

Será en el boletín mensual que la misma organización sindical publicaba desde marzo de 1913 titula- 
do, Mensajera del Sindicato de la aguja y similares de Ntra. Sra. de los Desamparados, en el que aparecerá por primera vez en junio de 1934, la referencia a una clase de enfermeras:

"La clase de enfermeras dirigida por el Dr. Moreu, ha venido funcionando durante todo el curso sin interrupción alguna y con gran interés por parte del profesor y con aprovechamiento de las alumnas; así lo han demostrado siendo un buen número de ellas las que se han examinado oficialmente en la Facultad de Medicina, obteniendo la calificación de notable y sobresaliente; se ha organizado este verano un cursillo breve para las que quieran examinarse en septiembre, y el curso complementario de ética profesional cristiana de la enfermera. Reciban nuestra felicitación, tanto el Profesor como las alumnas, por el éxito obtenido"'.

Este dato aislado encontrado en el Boletín publicado por el Sindicato femenino, es el que irremediablemente nos llevó a indagar en el Archivo de la Confederación Regional de los Sindicatos Valencianos, actualmente Obra Social Femenina de la Virgen de los Desamparados, para protección, asistencia y formación integral de la mujer que vive del trabajo, en adelante OSF, en busca de nuevos datos.

Esta fue la denominación con la que a partir de la ley de 26 de enero de 1942 sobre la Unidad Sindical del Estado tomaría el hasta entonces conocido como Sindicato Obrero de Trabajadoras de la Aguja fundado por Pérez Arnal en 1912. Su fin principal se asemejaba en gran medida al perseguido por la organización sindical pues sus estatutos establecieron como fin y objeto principal de la misma: "El fomento ya individual, ya organizado, permanente o transitorio, de toda actuación de protección, asistencia y de formación integral de la mujer que vive del trabajo, de cualquier profesión"5.

Además, de esta colaboración con la Central Nacional Sindical, la OSF quedaría integrada como obra auxiliar de la Acción Católica y en su carácter de obra auxiliar remitía listado completo de todos los miembros diocesanos que la integraban, así como la designación de un miembro que actuase como vocal en la Junta diocesana de Acción Católica. A cambio, la OSF, conservaba su autonomía propia y mantenía su dirección siempre que se mantuviese una coordinación y colaboración con los organismos oficiales ${ }^{6}$.

La organización de enfermeras existente en la OSF, data originalmente de 1932, así lo atestigua el fragmento referido a la fundación de esta sección en las memorias de la OSF:
"Un pequeño grupo de valientes enfermeras (conviene recordar que por aquel entonces acababa de estallar la República) que no se arredraron ante todas las dificultades que con motivo de aquél odioso régimen se presentaban a cada momento. Desde entonces hasta la fecha, (...) ha llevado una gran actividad en beneficio de las enfermeras componentes de dicha Agrupación que tiene por Patrona a la Virgen de la Salud"7.

La misma memoria referida en la nota anterior, habla de 279 hojas de solicitud de ingreso en la organización, y entre ellas, se contabilizaban 9 solicitudes de ingreso al gremio de enfermeras.

La Sección de enfermeras, quedaba oficialmente reorganizada en la OSF, a principios del curso 1940-41, y se constituía como una agremiación o hermandad que englobaba tanto a enfermeras tituladas como a aquellas que quisiesen recibir formación para acceder al título. Además de actuar como instrumento para la formación y preparación de las futuras enfermeras, tenía un carácter aglutinador que contemplaba otros aspectos relacionados con la formación moral y la protección laboral de las profesionales.

A continuación, reproducimos el fragmento relativo a la reorganización de la sección de enfermeras localizado en la memoria de los trabajos realizados en la OSF relativa a 1940 :

"Ha quedado constituida la sección de enfermeras, que cuenta en la actualidad con 70 socias. Esta asociación organizó al principio de curso las clases preparatorias para enfermeras dadas por los Dres. Moreu y Gallardo, matriculadas en dichas clases 40, también se tiene en proyecto la clínica de urgencia, que en breve funcionará para que se beneficien de ella todas las socias que lo deseen. En el segundo semestre del mismo año, la sección de enfermeras cuenta con 20 nuevas asociadas".

Esta Sección de enfermeras incluía socias enfermeras con título de Cruz Roja unas, otras con el de Sanidad Militar, otras con el de Puericultura, otras con el de Falange, y muchas otras con el de la Facultad de Medicina, ${ }^{9}$ lo que confirma la diversidad de posibilidades formativas que se contemplaban en el panorama nacional para poder optar al título de enfermera profesional.

La Orden Ministerial de 21 de mayo de 1941 que daba validez oficial a los estudios ofrecidos por Cruz Roja y otras instituciones similares, y cifraba en dos años la formación necesaria para la obtención del título de enfermera a través de un plan de estudios aprobado por la facultad de medicina correspondiente, 
constituiría una oportunidad de regulación profesional, y sería aprovechada por la Sección de enfermeras de la OSF para ofrecer cursos preparatorios que facilitarían la preparación de las futuras enfermeras para superar el examen oficial. "Acaban de comenzar hace unos días, las clases correspondientes al curso preparatorio de la escuela de enfermeras. En ellas se prepara a la futura enfermera para ingresar en los dos cursos que hoy existen para dicha carrera, siendo siete asignaturas las que en este curso preparatorio se dan. $A$ todas las que les interese seguir la profesión de enfermeras, pueden pasar los martes y los viernes a las siete y media por las oficinas del Gremio de enfermeras, y podrán obtener más detalles"10.

Como obra católica comprometida con la formación y promoción femenina, el cuidado de la vertiente moral y espiritual de las futuras enfermeras no quedaba desatendido y la oferta de cursillos de ética profesional, así como la participación en retiros espirituales o la celebración de misas de hermandad de cada gremio, sería una constante de la que se las memorias de actividades del curso también se harían eco.

"La formación moral y espiritual de nuestras asociadas, se ha llevado a cabo por medio de la Misa de la Hermandad mensual con meditaciones propias, ejercicios espirituales, conferencias de religión y moral, cursillos de ética profesional y fiesta religiosa anual propia de nuestra Agrupación, que como dijimos al principio de esta nota, está bajo la advocación de la Virgen de la Salud"11.

La formación práctica de las futuras enfermeras, se realizaba a través del gabinete de asistencia médica que la OSF ofrecía a sus afiliadas y familiares. El registro de inyectables administrados o las curas realizadas en el gabinete, confirman la actividad llevada a cabo por estas mujeres, puede observarse dicha actividad en la tabla número 3 de este mismo artículo.

La defensa profesional de las enfermeras se garantizaba a través de la organización de un Servicio domiciliario desde el cual, aquellos que tuviesen necesidad, podían reclamar sus servicios, acordando cuestiones laborales relativas a duración de la jornada o remuneración de la misma.

Tampoco hemos olvidado la protección y defensa profesional de nuestras compañeras, teniendo organizado con miras a lo primero, un servicio Domiciliario donde nuestras socias enfermeras, tienen un medio de ejercer la profesión remuneradamente. Dicho servicio está establecido de la siguiente forma: Servicio nocturno: de 10 de la noche a 7 de la mañana, 15 pesetas incluida la cena. Las horas de prolongación de servicio a petición de los interesados, se cotizan a 2 pesetas la hora ${ }^{12}$.

Tabla. 1. Número total de asociadas a la OSF desde 1939 a 1953.

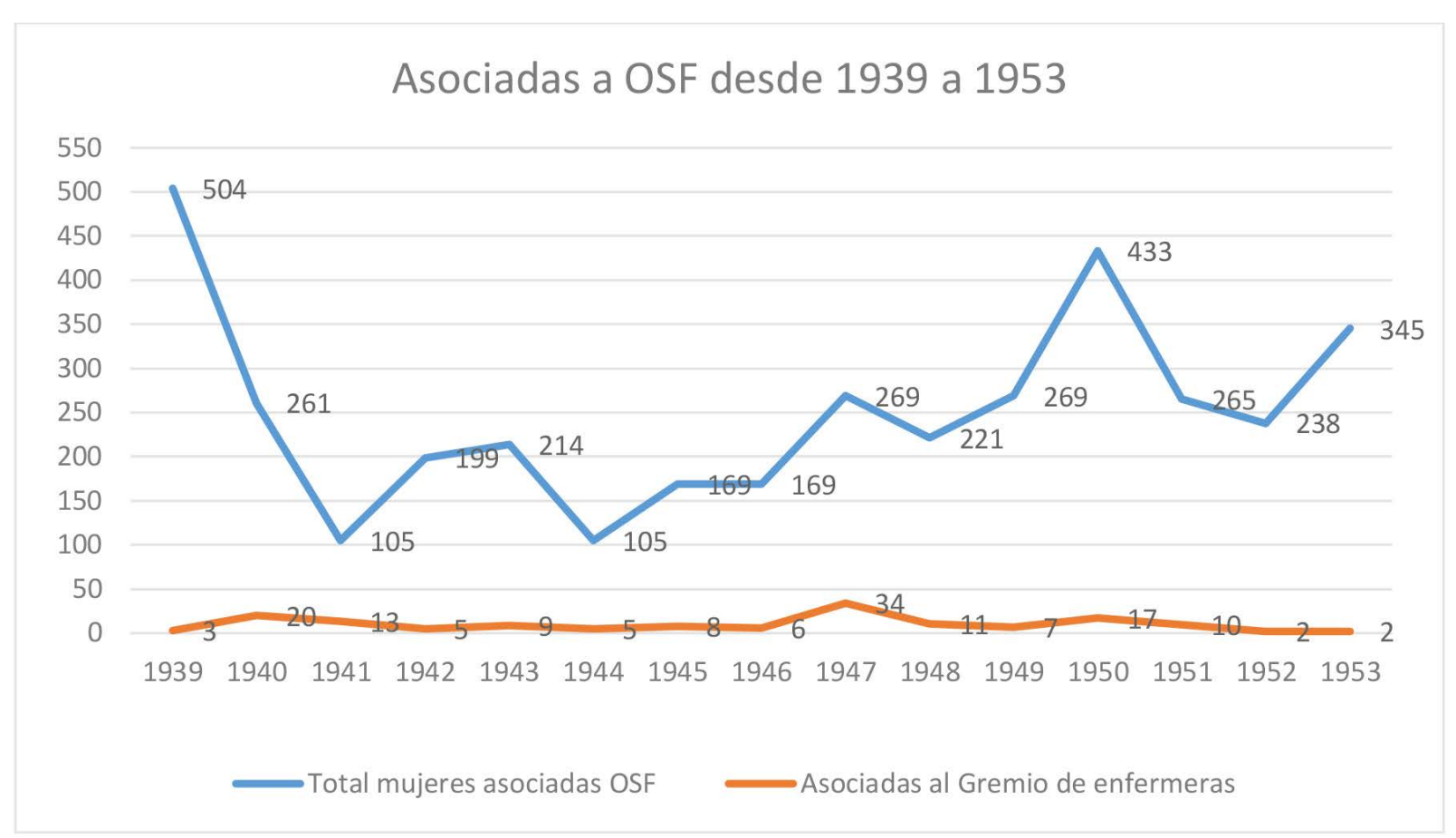


Tabla. 2. Número de asociadas al Gremio de enfermeras de la OSF desde 1939 a 1953.

\section{AFILIADAS AL GREMIO DE ENFERMERAS DE LA OSF DESDE 1939 A 1953}

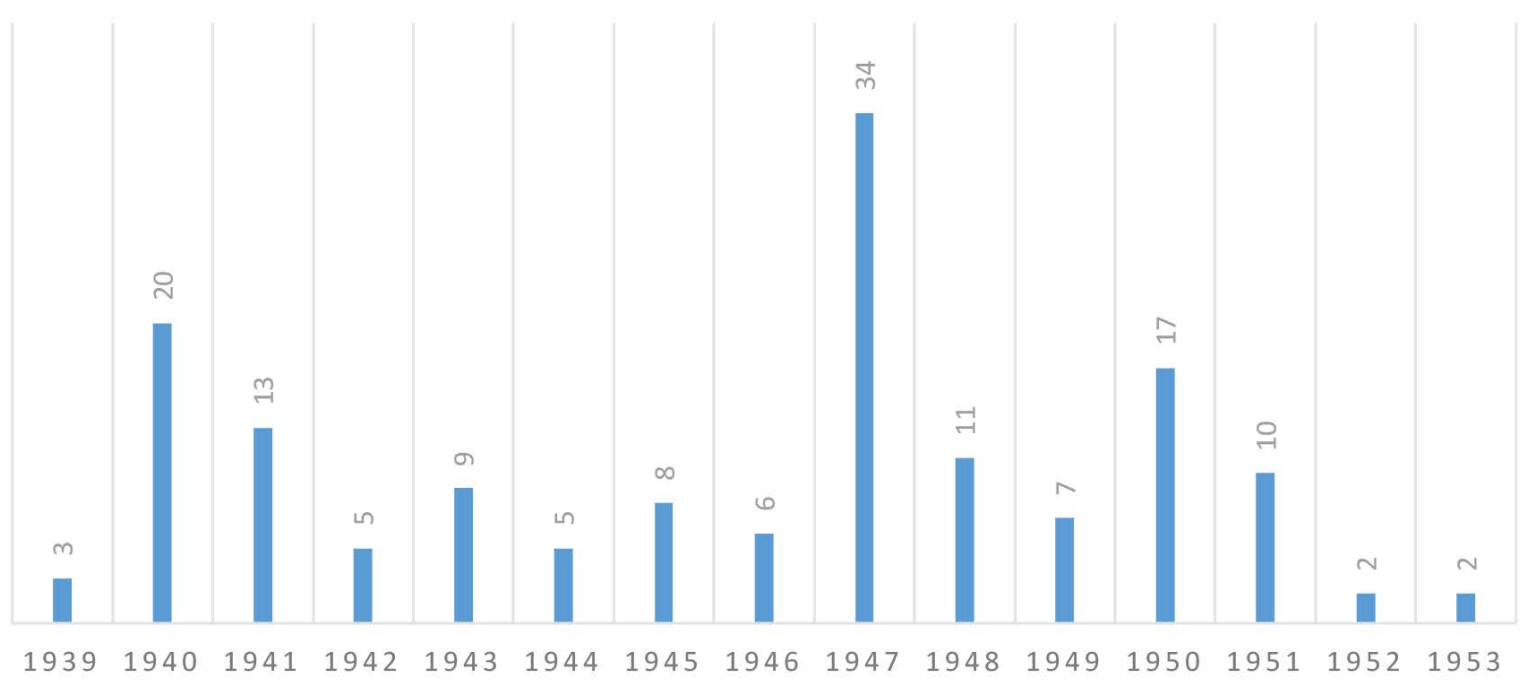

Tabla. 3 Actividades desempeñadas por las enfermeras de la OSF en el Gabinete de asistencia médica-

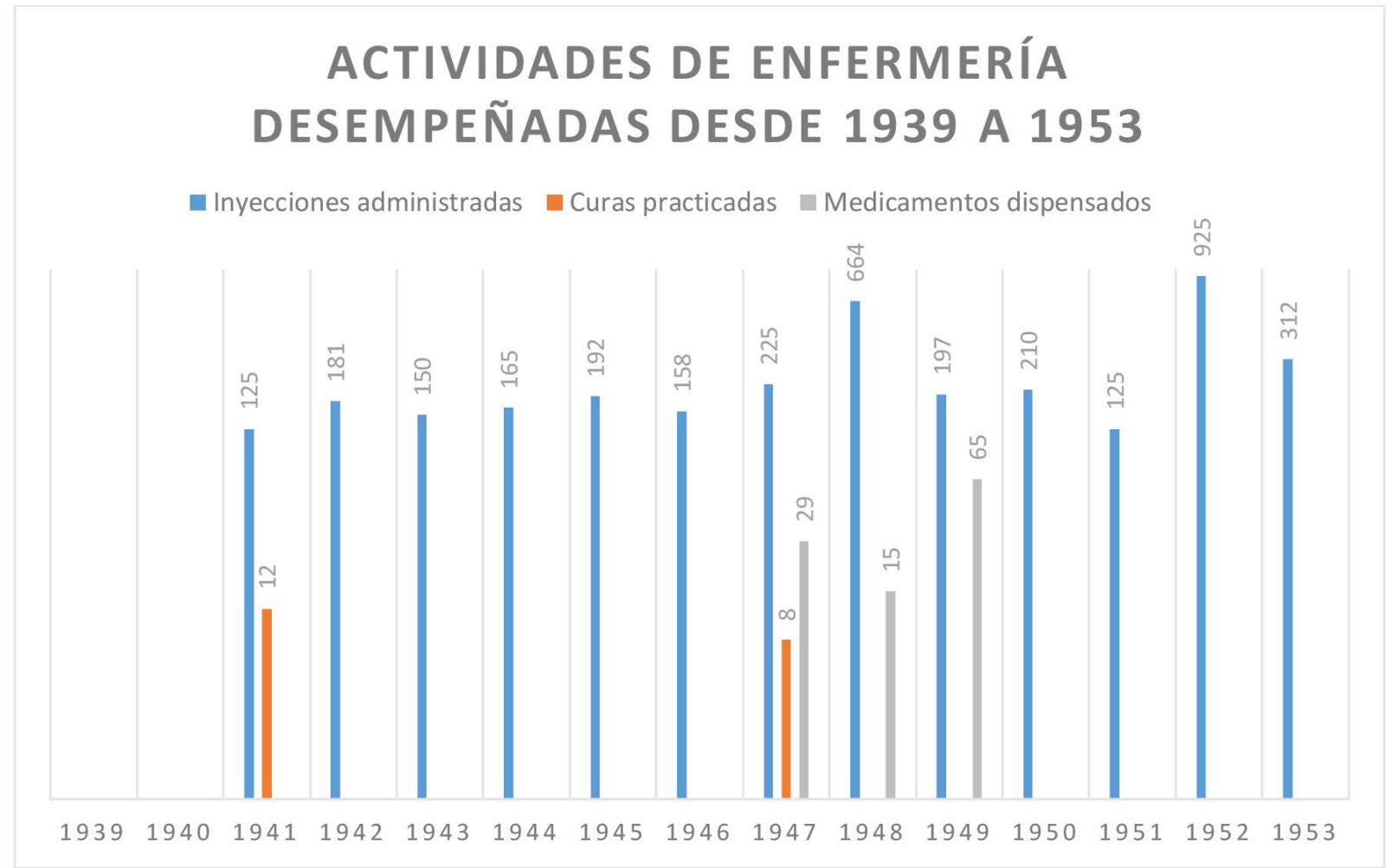


Los datos que confirman la actividad del gremio de enfermeras se recogen en las memorias de la OSF. Mostramos a continuación la evolución de esta agremiación profesional desde su reorganización en 1939 hasta 1953, año en el que se inaugura en Valencia la Escuela de enfermería de Ntra. Sra. de los Desamparados y se dictan las normas para la nueva organización de estudios de enfermería. Nos ayudamos de algunos gráficos que nos facilitan la comprensión de los datos de un modo más global. (Ver tablas 1 y 2 )

Dicha escuela, inaugurada en 1953, seguiría adaptándose a los continuos cambios acontecidos en la formación enfermera. La aprobación del Real Decreto $2128 / 1977$ de 23 de julio, sobre la integración en la universidad de las escuelas de ATS como Escuelas Universitarias, convertía a las escuelas existentes en las facultades en universitarias directamente, y las que dependiesen de instituciones privadas debían solicitarlo al Rector de la universidad a la que debían quedar adscritas. El 19 de octubre de1978, la Escuela de Enfermería de Ntra. Sra. de los Desamparados de Valencia, quedaba autorizada a matricular a los primeros alumnos, tanto hombres como mujeres, en la Escuela Universitaria de Enfermería de Ntra. Sra. de los Desamparados, adscrita desde entonces a la Universidad de Valencia. Más tarde, la escuela universitaria pasaría a depender de la Universidad Católica de Valencia, a partir de 2005. Con la nueva normativa de integración en el Espacio Europeo de Educación Superior, la Universidad Católica de Valencia, transformaría su Escuela Universitaria en Facultad de Enfermería de donde a partir de entonces egresarán enfermeros graduados, posibilitándose así la regulación de los estudios de post grado y ofreciendo a la enfermería, un desarrollo formativo completo, encaminado a la especialización en el ámbito académico y/o investigador.

\section{DISCUSIÓN}

La eclosión de iniciativas a favor de la promoción personal y profesional de la mujer, no surge como un fenómeno aislado o puntual en Valencia. Si allí se inauguraba un sindicato femenino protector de obreras y trabajadoras durante los primeros años del siglo XX, otras ciudades españolas sufrirían fenómenos de la misma índole. Tal es el caso de Madrid donde se fundó el primer sindicato femenino en 1909 con el nombre de Sindicato de Oficios Varios o el de Barcelona fundado en 1909 bajo el nombre de Sindicato de la Aguja. En Vitoria, a partir del Sindicato de la "blanca" fundado en 1909, surgirían nueve sindicatos femeninos a lo largo de los diez años siguientes. En 1913 se constituyó en Gijón el sindicato de sirvientas, el de la aguja y el de las cigarreras. Ciudades como Irún, Oviedo, Santander, Cádiz, Jerez de la Frontera, León, Montilla y Novelda también contarían con sindicatos para sus obreras trabajadoras en el sector textil. En 1918 se crearía el sindicato de modistas de la Vall d'Uxó, y en 1919 , en esta misma localidad, se inauguraba el sindicato de obreras de fábrica y el de sirvientas. En conjunto existían en España en 1921 unos 100 sindicatos católicos femeninos con un total de 20.000 obreras afiliadas (Benavides Gómez, 1978, p. 274).

Este sindicalismo de origen católico, surgiría con fuerza para reivindicar unas condiciones sociales y laborales dignas para un sector poblacional que no había sido tenido en cuenta hasta entonces (Luengo López, 2009, p. 95-120).

El nuevo marco civil y eclesiástico radicalmente distinto a la etapa anterior a la guerra civil, exigirá una adaptación legal de la situación del sindicato femenino valenciano que, bajo la denominación de Obra Social Femenina de Nuestra Señora de los Desamparados, seguirá trabajando por la promoción de la mujer. Tampoco la asociación o gremio de enfermeras inserto en esta Obra Social será un fenómeno aislado en el asociacionismo profesional enfermero de los años cuarenta, pues en Madrid, de la mano de su fundadora María de Madariaga y Alonso (19052001) se pondría en marcha una Hermandad Profesional de enfermeras cuyo objetivo comprendería la agrupación profesional de las enfermeras católicas, con la finalidad de alcanzar la mejora en el ámbito religioso, moral profesional, científico profesional y apostólico de sus componentes. (Chamorro Rebollo, 2015, p. 129)

La similitud de objetivos perseguidos entre las dos asociaciones profesionales propiciaría el encuentro de Doña Ana Pons Carda, responsable de la agrupación de enfermeras valenciana con la presidenta nacional de la Hermandad profesional Salus Infirmorum de Madrid, Doña María de Madariaga, con la finalidad de conseguir integrarse en esta Hermandad Nacional que en los años cincuenta estaría ya presente en varias ciudades españolas ${ }^{13}$.

El 15 de noviembre de 1949 quedaría constituida la Hermandad en Valencia, y la publicación periódica de la Hermandad Profesional difundiría la noticia.

Un año después de la puesta en marcha de la Hermandad en Valencia, el 26 de febrero de 1950, quedaría inaugurada en el número 4 de la calle Mosén Milà de Valencia, la sede social de la Hermandad Profesional en 
Fig. 1. Fuente: Revista Salus Infirmorum, Tomo I, Vol. XI, (1950) p. 5.

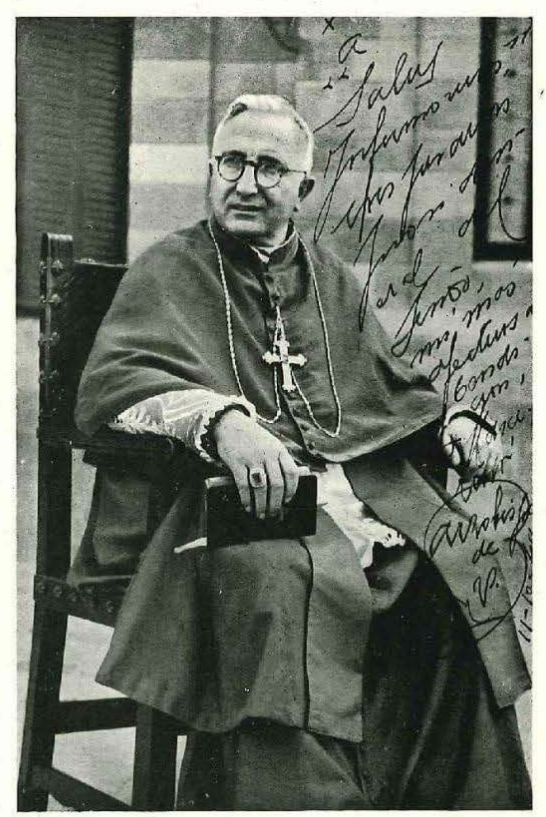

Valencia. El arzobispo diocesano Don Marcelino Olaechea, representantes de la sanidad valenciana y de la Acción Católica, se darían cita en el acto inaugural.

Salus Infirmorum, tuvo un papel fundamental en el desarrollo de la formación enfermera en los años cuarenta, aportando la experiencia acumulada de diez años en la formación y participando de forma activa en la Comisión Ministerial para la reforma de los estudios de enfermera. Entre sus contribuciones más importantes en esta comisión, destacar que elevó a tres años la formación en régimen de internado de las alumnas, luchó por la incorporación de materias como la Religión y la Moral en los planes de estudios, y llevó al ámbito extra hospitalario la formación práctica de las alumnas (Chamorro Rebollo 2016, p. 388).

La presencia de esta Hermandad Profesional de enfermeras en Valencia fue crucial en un momento clave para la historia de la profesión, la publicación de la orden de 4 de agosto de 1953 que dictaba las normas para acceder a los estudios de enfermera (BOE 244, p. 5258), fue posible gracias a la intervención de una pequeña agrupación surgida desde las filas del aso-
Hermandad Profesional dle Enfermeras Españolas. "SALUIS INFIRMORUM"

Primera reunión oclchrada en Valencia, en el Palauio Arzobispal, bajo la presidencia del Excmo. y Rvdmo. Sr. Arzobispo de la Diócesis, Doctor D. Marcelino Olacchea y Loizaga, y de los miembros elegidos para formar la junla de Gobierno de esta. Ilermandad.

PROGRAMA

1. Presentación de la Junta de Gobierno y Hermanas Cunscjoras,

2. Lectura del Reglamento de la Hermanda

- Entrega de los Boletines de inscripción.

4. Exhortarión del Exemo. Sr. Arzobispo.

- Visita al Sugrario en la Ciapilla de Palacio.

JUNTA DE GOBIERNO

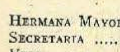

SECRETA

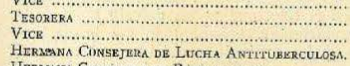

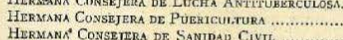

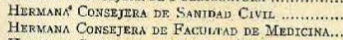

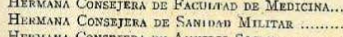

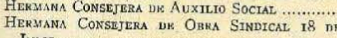

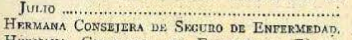

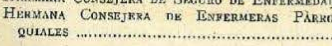

VALENCIA DEL CID, 15 de noviembre de 1949

ciacionismo confesional de principios de siglo. Este vínculo entre asociaciones profesionales hasta ahora desconocido, evidencia el compromiso de un pequeño grupo de mujeres con la promoción profesional de la enfermería en el ámbito local.

\section{CONCLUSIONES}

1. Las condiciones de pobreza, hambre, insalubridad o hacinamiento en las que queda el país tras el conflicto bélico, exigen una actuación urgente por parte del nuevo gobierno estatal.

2. Las medidas eugenésicas llevadas a cabo durante los primeros años de la posguerra encontrarán en la mujer el instrumento idóneo para vehiculizar estas políticas de higiene, salubridad y natalidad a favor del engrandecimiento de la Patria.

3. El oficio de enfermera se consolida como pieza esencial en la medicina social del franquismo, aunque existe gran variedad de figuras profesionales con cometidos de gran similitud. 
4. Las nuevas órdenes legislativas posibilitarán a instituciones privadas formar a futuras enfermeras siempre que los planes de estudios se ajusten a los planes aprobados por las facultades de medicina.

5. Desde el seno de la Acción Católica, única organización permitida al margen de la influencia directa del gobierno, se organizará una Hermandad profesional de enfermeras a nivel nacional que, bajo el nombre de Salus Infirmorum, ofrecerá a sus integrantes una formación técnica, moral y profesional basada en los principios del humanismo cristiano.

6. En el caso valenciano, alentado por los principios del catolicismo social, surge una iniciativa a favor de la promoción social, laboral y profesional de la mujer, el Sindicato obrero de trabajadoras de la Aguja y similares, bajo la protección de Nuestra Señora de los Desamparados.

7. Con el nuevo ordenamiento jurídico propuesto durante el franquismo el sindicato adoptará una nueva

\section{NOTAS AL FINAL}

1 Auxilio Social: Normas y orientaciones para delegados provinciales: Primer Congreso organizado por la Delegación Nacional de "Auxilio Social", 13-18 de septiembre 1937, Valladolid, 1937, p. 33

2 Estatuto del Sindicato de trabajadoras de la aguja y similares de la Virgen de los Desamparados, Valencia, Tipografía Moderna, AC. De M. Gimeno, 1916, art. 1 y 2, p.3.

3 Estatuto del Sindicato de trabajadoras de la aguja y similares de la Virgen de los Desamparados, Valencia, Tipografía Moderna, AC. De M. Gimeno, 1916, art. 44, p.14.

4 La Mensajera de la Confederación Regional de Sindicatos Femeninos de Ntra. Sra. de los Desamparados, n. 153 (juniojulio 1934

5 El decreto de erección del Reglamento de la OSF, fecha de 27 de febrero de 1940, un año más tarde, el 22 de agosto de 1941 el arzobispado de Valencia aprobaría el Reglamento orgánico de la OSF. Reglamento OSF de la Virgen de los Desamparados para protección, asistencia y formación de la mujer que vive del trabajo, Archivo Obra Social Femenina de Valencia, (AOSFV).

6 "Bases para un proyecto de fusión de la OSF con AC," Archivo Metropolitano Arzobispado de Valencia (AAV), Documentación perteneciente a la causa de beatificación de Don Manuel Pérez, Arnal, sin catalogar.

7 Resumen de la organización de enfermeras existente en la OSF de Valencia del Cid. Valencia, 12 de noviembre de 1947, Archivo del Arzobispado de Valencia (AAV), Documentación denominación y la OSF quedará relegada a la categoría de centro colaborador y de apoyo a la única organización sindical permitida, a la vez que integrada en calidad de obra auxiliar en la Acción Católica diocesana.

8. La actual OSF, contará con una agrupación de enfermeras desde 1934 que tras el conflicto bélico continuará con su labor de formación y promoción de las enfermeras en el ámbito local.

9. En su lucha por la promoción personal y profesional de la mujer, la responsable de la agrupación local de enfermeras de la OSF procurará trasladar el modelo de Salus Infirmorum a Valencia consiguiéndolo en 1949.

9. A nivel local la agrupación de enfermeras impulsada por la OSF de Valencia y a nivel nacional la Hermandad profesional Salus Infirmorum, contribuirán a la formación personal y profesional de las enfermeras españolas en un periodo de importantes transformaciones para la profesión. perteneciente a la causa de beatificación de Don Manuel Pérez, Arnal, sin catalogar.

8 "Memoria de los trabajos realizados en la OSF de Ntra. Sra. de los Desamparados en el primer semestre de 1940," AOS$\mathrm{FV}$, sin catalogar.

9 "Resumen de la organización de enfermeras existente en la OSF de Valencia del Cid. Valencia, 12 de noviembre de 1947," AAV, Documentación perteneciente a la causa de beatificación de Don Manuel Pérez, Arnal, sin catalogar.

10 "Memoria de los trabajos realizados en la OSF de Ntra. Sra. de los Desamparados segundo semestre de 1942," AOSFV, sin catalogar.

11 "Resumen de la organización de enfermeras existente en la OSF de Valencia del Cid. Valencia, 12 de noviembre de 1947," AAV, Documentación perteneciente a la causa de beatificación de Don Manuel Pérez, Arnal, sin catalogar.

12 Resumen de la organización de enfermeras existente en la OSF de Valencia del Cid. Valencia, 12 de noviembre de 1947," AAV, Documentación perteneciente a la causa de beatificación de Don Manuel Pérez, Arnal, sin catalogar.

13 Doña Ana Pons Carda, responsable de la agrupación de enfermeras en la OSF, dirige una carta al arzobispo de la diócesis Don Marcelino Olaechea y Loizaga con fecha de 25 de agosto de 1949 en la que le expone la necesidad de trasladar la Hermandad de enfermeras Salus Infirmorum a Valencia, y la necesidad de ponerse en contacto con María de Madariaga, máxima responsable de la Hermandad Nacional. Archivo Arzobispado de Valencia, (AAV) Documentación perteneciente a la causa de beatificación de Don Manuel Pérez, Arnal, sin catalogar. 


\section{BIBLIOGRAFÍA}

Agulló Díaz, Maria Carmen (1999), “Azúl y rosa. Franquismo y educación femenina". En: Mayordomo, Alejandro (coord.), Estudios sobre la política educativa durante el franquismo, Valencia, Martín impresores, 1999, pp.243-303. 251.

Arbiza Villalonga, Mercedes (2000), "La cuestión social como cuestión de género. Feminidad y trabajo en España (18601930)" Historia contemporánea, 21, pp. 395- 458.

Benavides Gómez, Domingo (1978), Democracia y cristianismo en la España de la Restauración 1875-1931. Madrid, Ed. Nacional, pp. 274.

Bernabeu Mestre, Josep; Gascón Pérez, Encarna (1999), Historia de la enfermería de salud pública en España (18601977), Alicante, Publicaciones de la Universidad de Alicante, pp.102-109.

Blasco Herranz, Inmaculada (2008) "Mujeres y "cuestión social" en el catolicismo social español: los significados de la "obrera", Arenal, 15 (2), pp. 236-268, p.242.

Camaño-Puig, Ramón; Forero Rincón, Olga. (1998-1999), “Escuelas de enfermería: de la escasez a la abundancia...Su evolución histórica, (1857-1977)," Híades, Revista de Historia de la enfermería, 5-6, pp. 155-167, p.161.

Cantero González, María Lourdes; Hernández Conesa, Juana María; Beneit Montesinos, Juan Vicente (2010), "La formación enfermera durante el franquismo". En: Formación e identidad enfermera en el franquismo, Murcia, Diego Marín librero editor, pp. 255-274.

Capel Martínez, Rosa María (1986), El trabajo y la educación de la mujer en España. (1900-1930) Madrid, Ministerio de cultura Instituto de la mujer, pp. 161-163.

Chamorro Rebollo, Elena (2016), La influencia de María de Madariaga y de Salus Infirmorum en la Enfermería española en la última mitad del siglo XX, Tesis doctoral, José Siles-González (dir.) Alicante, Universidad de Alicante, pp. 122-132.

Chaves Palacios, José (2004), “Desarrollo tecnológico en la primera Revolución Industrial", Norba. Revista de Historia, 17, pp. 93-109.

Cayuela Sánchez, Salvador (2014), Por la grandeza de la patria, la biopolítica en la España de Franco (1939-1975), Madrid, Fondo de Cultura económica de España, pp. 202-203.

Domínguez Isabel, Patricia; Espina Jerez, Blanca; Gómez Cantarino, Sagrario; Elena Hernández, Azucena; De Dios Aguado, Mercedes; Pina Queirós, Paulo Joaquim (2019), “Organización de los cuidados de enfermería en la Guerra Civil española (1936-1939): un abordaje histórico," Cultura de Cuidados, 53 pp. 77-86.

Escudero Gutierrez, Antonio (1999), “Dos puntualizaciones sobre la historia de la siderurgia española entre 1880 y 1930 ", Revista de Historia Industrial, 15, pp. 191-200.

España, "Orden Ministerial de 21 de mayo de 1941 sobre condiciones y estudios necesarios para la obtención del título de enfermera", Boletín Oficial del Estado, 148, p. 3811.

España, "Ley de 3 de enero de 1942 por la que se crea el Cuerpo de enfermeras de Falange Española Tradicionalista y de las JONS", Boletín Oficial del Estado, 13, p. 244.
España, "Orden de 4 de agosto de 1953 por la que se dictan las normas para la organización de los estudios de enfermera", Boletín Oficial del Estado, 244, p. 5258-52560.

España, “Orden de 4 de julio de 1955 por la que se aprueba el programa formativo de los Ayudantes Técnicos Sanitarios (A.T.S.)", Boletín Oficial del Estado, 214, p. 4751-4752.

Fernández Jiménez, María Antonia (2008), Pilar Primo de Rivera, el falangismo femenino, Madrid, Síntesis, p. 231.

García Ferrandis, Xavier (2013), “Aspectos epidemiológico- asistenciales de la tuberculosis en Valencia durante la guerra civil española y la posguerra inmediata (1936-1941)", Llull: Revista de la Sociedad Española de Historia de las Ciencias y las técnicas, Zaragoza, 36 (77) pp. 13-34.

León XIII (1891), Rerum Novarum, [en línea], disponible en http://www.vatican.va/content/leo-xiii/es/encyclicals/ documents/hf_I-xiii_enc_15051891_rerum-novarum.html [consultado el 22/1/2020].

López Vallecillo, María (2016), "Relevancia de la mujer en el bando nacional de la Guerra Civil española: las enfermeras", Memoria y civilización, Pamplona, 19, pp. 419-439.

Luengo López, Jordi (2009), "El sindicato de la Aguja. Asociacionismo femenino en Valencia de la Gran Guerra (19141918)", Cuestiones de género: de la igualdad y la diferencia, 4, pp. $95-120$

Monlleó Peris, Rosa (2004), "Señoritas y obreras bajo la tutela de la Iglesia", Millars: Espai i historia, 27, pp. 123-164. 133.

Montero García, Feliciano (1984), “El primer catolicismo social en España. Estado de la cuestión", Studia Histórica. Historia contemporánea, 2, pp.185-192, 187-188.

Nash, Mary (2000), “Maternidad, maternología y reforma eugénica en España, 1900-1939", en Duby, Georges; Perrot, Michelle (dir.) Historia de las mujeres en Occidente. Madrid, Taurus Minor, Vol. V, pp. 687-692. 693.

Palacio Lis, Irene (2003), Madres ignorantes: madres culpables. Adoctrinamiento y divulgación materno- infantil en la primera mitad del siglo XX, Valencia, Martín impresores, pp.196242.

Palacio Lis, Irene; Ruiz Rodrigo, Cándido (1990), “Educación de la mujer obrera en Valencia. Del Sindicato de la Aguja a la Obra Social Femenina". En: VI Coloquio de Historia de la Educación. Mujer y Educación en España (1868-1975), Santiago de Compostela, pp. 650-662. p. 651.

Ramón Fernández, Francisca (2001), Historia del Sindicato de la Aguja y similares. Obra Social Femenina de la Virgen de los Desamparados, Nàquera, Excmo. Ayto. de Nàquera, p. 87.

Raveux Oliver (2005), "Los fabricantes del algodón de Barcelona (1833-1844). Estrategias empresariales en la modernización de un distrito industrial", Revista de Historia Industrial, 28, pp. 157-184.

Sánchez Blanco, Laura (2010), "Las políticas demográficas italiana y española: L’Opera Nazionale per la protezione della Maternità e dell'Infanzia y La Obra Nacional Sindicalista de Protección a la Madre y el Niño". En: Boota, Patrizia (coord.), Actas del XVII Congreso de la Asociación Internacional de Hispanistas: Rumbos del hispanismo en el umbral del cin- 
cuentenario de la AlH, Roma, Bagatto libri, Vol. VII, pp. 227236.

Scott, Joan Wallach (1993), "La mujer trabajadora en el siglo XIX". En: Duby, G. ; Perrot T, M. (dirs.), Historia de las mujeres. El siglo XIX, IV, Madrid, Taurus, pp. 427-461.

Siles González, José (2008), “La formación de los profesionales de la enfermería tras la Guerra Civil: del nacional-catolicis- mo y la Sección Femenina a la etapa universitaria". En: Siles González, José (ed.), Historia de la enfermería, Alicante, Editorial Agua Clara, pp. 284- 290.

Teixidor de Otto, María Jesús; Hernández Soriano, Teresa (1998), "El trabajo de la mujer en las labores del tabaco. La fábrica de València (1887-1914)", Cuadernos de geografía, 64, pp.339-354. 